\title{
Guest editorial: special issue on ECIR 2020
}

\author{
Joemon M. Jose ${ }^{1} \cdot$ Emine Yilmaz $^{2} \cdot$ João Magalhães ${ }^{3} \cdot$ Pablo Castells $^{4}$
}

Received: 6 January 2021 / Accepted: 7 January 2021 / Published online: 28 January 2021

(c) The Author(s), under exclusive licence to Springer Nature B.V. part of Springer Nature 2021

The European Conference on Information Retrieval (ECIR) is the prime European forum for the presentation of original research in the field of Information Retrieval. The 42nd European Conference on Information Retrieval (ECIR) was held in Lisbon, Portugal, during April 14-17, 2020, and brought together hundreds of researchers from all over the world. Given the ranging pandemic, ECIR 2020 was the first online IR event. This is a special Issue for the selected papers from the ECIR 2020 conference.

There are three papers in this special issue. The paper entitled, "A Comparison of Automatic Boolean Query Formulation for Systematic Reviews", by Harrisen Scells, Guido Zuccon and Bevan Koopman presents a study on comparing automatic Boolean query formulations for systematic reviews. Screening literature is an important task that constitute a significant amount of time and effort in the systematic review creation process. To complete this task, a Boolean query is used, as it allows for complete control over the search system and enables the explicit encapsulation of the information need of the underlying research question into the query syntax. Even for expert searchers, query formulation for systematic reviews can be difficult and lengthy: especially when dealing with areas of medicine that they may not be knowledgeable about. Two computational adaptations of methods information specialists use to formulate Boolean queries were compared in this work. This work helps to inform those building search tools that assist with systematic review construction.

The paper entitled, "Improving question answering for event-focused questions in temporal collections of news articles", by Jiexin Wang, Adam Jatowt, Michael Färber, and Masatoshi Yoshikawa presents a study on the question answering for event-focused collections on temporal news archives. Temporal collections of news articles contain numerous accurate and time-aligned articles, which offer immense value to our society, helping users to know details of events that occurred at specific time points in the past. Authors make use of the observation that questions on news archives are usually related to specific events and show strong temporal aspects. Hence, they propose a large-scale question answering model

Joemon M. Jose

Joemon.Jose@glasgow.ac.uk

1 University of Glasgow, Glasgow, UK

2 University College London, London, UK

3 Universidade NOVA de Lisboa, Lisbon, Portugal

4 Universidad Autónoma de Madrid, Madrid, Spain 
designed specifically for long-term news article collections, with an additional module for re-ranking articles by using temporal information from different perspectives.

The paper entitled, "Review helpfulness evaluation and recommendation based on an attention model of customer expectation" by Xianshan Qu, Xiaopeng Li, Csilla Farkas, John Rose presents a model to predict review helpfulness automatically. It is essential to identify helpful reviews on online platforms, given the large number of reviews and wide range of quality of these reviews. This work is inspired by the observation that a customer's expectation of a review can be affected by review sentiment and the degree to which the customer is aware of pertinent product information. Consequently, a customer may pay more attention to that specific content of a review which contributes more to its helpfulness from their perspective. To model such customer expectations and capture important information from a review text, they propose a novel neural network which leverages review sentiment and product information.

The BCS IRSG committee and the Springer Information Retrieval Journal editorial board, has formed a memorandum of agreement for creating special issues of the selected ECIR conference papers. This year we made the first attempt towards this goal. Given the pandemic raging in the community, it was a challenging process. We have invited ten paper authors of the ECIR 2020 conference and finally selected three papers from the submissions. We thank the reviewers for the special issue and thank all the contributors submitted to the special issue.

Publisher's Note Springer Nature remains neutral with regard to jurisdictional claims in published maps and institutional affiliations. 